\title{
The Evolution of the Nervous System: Invertebrates vs. Vertebrates a useful Instrument and Model to Research New Pharmacological Strategies in some Human Neurodegenerative Conditions
}

\author{
Mauro Luisetto ${ }^{1 *}$, G Ibrahim², Oleg latyschev ${ }^{3}$ and Muhammad Akram \\ ${ }^{1}$ Branch Natural Science, Applied Pharmacologist, Italy \\ ${ }^{2}$ Department of Zoology, Alexandria University, Egypt \\ ${ }^{3}$ President IMA Academy, India \\ ${ }^{4}$ Department of Eastern Medicine, Government College University, Pakistan
}

*Corresponding author: Mauro Luisetto, IMA academy, branch Natural Science, applied pharmacologist, Italy.

To Cite This Article: Mauro Luisetto. Physiology of The Dependence on Chemical And Psychogenic Psychoactive Factors. Am J Biomed Sci \& Res. 2019 - 5(5). AJBSR.MS.ID.000960. DOI: 10.34297/AJBSR.2019.05.000960.

Received: 眥 October 11, 2019; Published: 战 October 16, 2019

\begin{abstract}
Nervous System Functions, Evolutive Pattern, and Relationship with Some Neurodegenerative Condition:

The nervous system directs and coordinates our movements. It receives stimuli from the environment around us and from all our internal organs. It interprets these stimuli and elaborates responses, which are transmitted to muscles or glands. To compare the nervous systems of invertebrates to vertebrates and between the various vertebrates.

Can be a useful instrument in field of neuroscience, molecular biology, forensic science, and pharmacology, regenerative medicine.

Why in example some neuronal circuits in superior vertebrates cross themselves like decussatio pyramid or in optical chiasm?

And why in brain vs spinal cord grey matter and white matter are inverse (opposite)?

And this fact makes possible to verify relationship between degenerative pathology of cortex vs spinal cord under an evolutive approach.

And this can be related also to the "brain and spinal cord wasting system" functionality?

Is universally known that different neurodegenerative disease involved different CNS parts: cortex (DA), PD (basal nuclei), ALS spinal cord preferentially.

And this is an interesting fact: a same organ (CNS) but whit different neuronal sensibility to the damages like neurodegeneration.

Is universally known that in superior vertebrates $\mathrm{AD}$ is a common disease in animals whit medium -high cognitive functions (frontal cortical and other) and not only a human condition.

An evolutionary approach makes possible to translate to applied research crucial information to better Explore new therapeutic strategies for some neurodegenerative pathology.

Keywords: Nervous Systems; Vertebrates; Invertebrates; Neuroscience; Evolutive; Research; Neurodegenerative; Forensic Sciences; Pharmacology Research; Regenerative Medicine
\end{abstract}

\section{Introduction}

Is possible to start this work with a question: observing various animals is possible to see the same incidence of neurodegenerative disease?
DA is not only a human pathology, other species present this condition (cats, dogs and other).

Obviously is superior vertebrate's whit high cortical - cognitive evolution. 
If this function is less physiologically - anatomically developed is difficult that this animal can show DA.

Is a Paradox, but in evolutive pattern of superior vertebrates something goes wrong?

The different vulnerability of CNS neurons in the different place of the brain or spinal cord seem to tell us that in evolution the new structure added to the oldest are more vulnerable.

For this reason, is crucial to set the neurodegenerative disease under an evolutionary

Approach.

A more complex nervous system (invertebrates vs vertebrates) create a very different organ with advantages but also disadvantages.

From university lessons, adapted: The Evolution of the Nervous System (by Gaber Ibrahim)

In the lower multicellular animals, such as porifers or sponges, there is no rudiment of nerves. We begin to see neurons, cells that conduct nerve stimuli, in coelenterates. In cnidarian polyps these cells appear scattered throughout the body, forming a network without much organization. There is no nerve center in these animals that runs this network. Each external stimulus acting on a point on the body is accompanied by a merely local response, determining a nerve impulse that propagates with decreasing intensity as it moves away from the stimulus's starting point. Cnidaria have a diffuse nervous system.

\section{Cnidarian Phylum (corals, anemones, hydras and jellyfish)}

The more primitive Porifers (sponges) do not have a nervous system. In Cnidaria, there is a disordered network of neurons. And if a nerve pulse is triggered in one of them, it is transmitted to all cells that communicate with it through synapses, and from these to others, resulting in poorly elaborated responses - such as "pulsating" movements in living water when it's swimming. It is the most primitive type of nervous system, called Diffuse Nervous System.

\section{In Flatworm Worms (such as planar worms, for example)}

Neurons associate together to form nerve threads attached to some structures - the nerve ganglia in the head. These ganglia already represent precarious nerve centers in coordinating body activities. In each ganglion there is a higher concentration of neurons.

The ganglionic nervous system begins to perfect in the annelids. In them, there is a larger conglomeration of neurons in the head, forming the cerebroid ganglia, which play a primitive brain role in commanding the other ganglia. From the cerebroid ganglia arise the periesophageal ganglia, which relate to a double ventral ganglionic nerve chain. Along this chain there are a pair of ganglia for each body segment. These ganglia also have marked autonomy over the specific activities of the surrounding body area.

\section{In Annelids}

Notwithstanding the presence of cerebroid ganglia, the pairs of ganglia along the ventral nerve chain have a great deal of autonomy, so a worm, even after being cut in half, continues to move the two pieces apart.

The cerebroid ganglia are even more developed in arthropods, especially insects.

In bilateral symmetry invertebrates (Platelminths, Nematelmints, Annelids, Molluscs and Arthropods) the nervous system is in the ventral region of the body and is organized as one or more longitudinal nerve cords presenting two or more nerve ganglia, whether functioning as command centers along its length. In the possessors of many nerve ganglia, those in the anterior region-cerebroid ganglia are more developed and function as a rudimentary brain that controls the other ganglia. This type of nervous organization is called the ganglionic nervous system.

\section{In Mollusca}

The nervous system is centralized and ganglionic, with three parts of nerve ganglia from which nerves go to different parts of the body. Sensory, visual, tactile, chemoreceptor and balance structures are present. The cephalopods have a large cerebroid ganglion that resembles the brain of vertebrates.

\section{In gastropods}

The nervous system consists of a set of ganglia and cords that are distributed throughout the body and innervate the different organs. The set of sensory organs comprises eyes, tentacles, asphradium and statocysts. The eyes, in the most primitive forms, are located at the ends of the tentacles and consist of simple depressions containing pigment and photoreceptor cells. In more advanced gastropods, depression closes, and a cornea and a lens are distinguished. The tentacles have eyes and tactile and chemoreceptor cells. Statocysts are important sensory cells for balance. Available only in species with gills, appears to function as an olfactory and chemoreceptor organ.

Take a close note: The ganglionic nervous system, which characterizes invertebrates, has its double chain of lymph nodes arranged ventrally in the animal, that is, running along the ventral surface of the body. This system is in stark contrast to the vertebrate brain-spinal nervous system that we will see next. The cerebrospinal nervous system is in the dorsal position, descending from the head along the back of the animal [1].

\section{The Cerebrospinal Nervous System}

In vertebrates (fish, amphibians, reptiles, birds and mammals), the nervous system is well developed and is classified as a 
cerebrospinal nervous system. It is made up of a "thirst" - the CNS (central nervous system) - and a network of nerves that break out and distribute throughout the body - the peripheral nervous system.

\section{Vertebrates}

4.1.1.The Central nervous system: The CNS is formed by the brain and spinal cord. The brain, in turn, comprises the following portions: brain, cerebellum, protuberance (pons or menencephalon) and bulb.

In the lower vertebrates, from fish to birds, the cerebral hemispheres have a smooth surface. Such animals are considered diencephalon (smooth brain). In mammals, however, grooves and circumvolutions appear, giving the brain a surface full of undulations. For this reason, mammals are called gyrencephalon (brain with turns or curves). This transformation brought a great advantage for mammals: At the same volume, a circumvoluted brain has a considerably larger surface than if it had smooth hemispheres. As it is on the surface of the brain (cerebral cortex, with gray matter) that lie the bodies of neurons, the more grooves and convolutions the brain has, the larger its cortex, the larger the number of neurons, and thus the more efficient and improved it is.

The gray matter is placed on the surface of the brain and is where the bodies of neurons accumulate. It is in them that the information is stored, the senses are perceived, the data obtained from external stimuli are "processed". Also, from the neurons depart the orders for muscle contractions or for glandular secretions etc. This superficial area is the cerebral cortex. It has the greatest importance in the degree of development of a species.

The cerebral cortex is all divided into zones, like a map. Each area (some small, some large) represents a nerve center. Nervous centers are numerous throughout the brain, such as the centers of sight, hearing, smell, taste, pain, hunger, cough, tickling, anger, motor coordination (this is very wide and subdivides into areas corresponding to the various points of the body), the visual association for reading, in addition to the centers of respiratory, cardiac regulation, the thermoregulatory center, etc. The cortex is, as it turns out, the "seat" of control of conscious and unconscious acts as well as intelligence.

The brain of a crocodile is, of course, larger than a brain of a mouse. However, the crocodile, as a reptile, is lissencephalon, while the mouse, as a mammal, is a gyrencephalon. Therefore, the extension of the cerebral cortex of the mouse is larger than that of the crocodile, justifying greater rodent intelligence. That is why, in circuses, animal shows predominantly exhibit mammals.

In the deepest region of the brain lies the white mass. In it, there are practically no bodies of neurons, but only their branches (dentites and axons).

The cerebellum, pons, and bulb are also very important because they enclose nerve centers that regulate various functions of relevant role. Breath and temperature controls are in the bulb. Control of body balance is in the cerebellum.

Aside from the brain, the remainder of the CNS consists of the spinal cord (or spinal cord). It is a long cord of nervous structure that runs along the dorsum inside the spinal canal. It is therefore protected to its full extent by the spine. In spinal cord the gray mass (as opposed to the brain) is in the center and the white mass in the periphery.

White matter is buried deep in the brain and the gray matter is mostly found on the brain's - cortex.

The spinal cord that transmits nerve impulses to and from the rest of the body, has an opposite arrangement:

gray matter at its core with insulating white matter on the outside.

The brain white matter is made up by axon tracts, the long, spindly appendages of some brain cells.

These tracts transmit the electrical signals that the brain neurons, to communicate.

They're wrapped in a fatty- layer named myelin, this insulates axons, allows them to conduct signals in very quickly way, much like rubber insulation does for electrical wires.

The type of fat in myelin makes it seem white.

Gray matter is mostly neuron cell bodies and non-neuron brain the cells named glial cells, that provide nutrients and energy to the neurons.

They help in the transport of the glucose into the brain, clean the brain of excess chemicals and may even affect the intensity of the neuron' s- communications systems.

In central nervous system there are a mix of cell types present in both gray and white matter.

\section{a. Gray Matter Contains:}

- $\quad$ Neurons

- $\quad$ Axon tracts

- $\quad$ Glial cells

- Capillary blood vessels

- $\quad$ Neuropil-mix of dendrites, un-myelinated axons, glia

b. White Matter contains:

- $\quad$ Oligodendrocytes-glial cells which produce myelin

- $\quad$ Astrocytes

\section{Function of Gray Matter}

a) Gray matter-heavy- brain regions include those that control muscular /sensory activity. 
b) The outer layer of the brain, the cerebral cortex, consists of columns of gray matter neurons, with white matter located underneath.

c) This area is essential to many facets of higher learning functions, attention, memory, and thought.

d) The cerebellum is essential for motor control/ coordination/ and precision of movements.

\section{Function of White Matter}

e) Neuron-rich brain regions join themselves by the rich veins of axonal connections contained within white matter

f) The white fatty myelin is essential to its function - myelin insulates axons, letting the signal within travel far faster, this make possible the nerve cell function: essential to normal motor and sensory function (Figure $1 \& 2$ ).
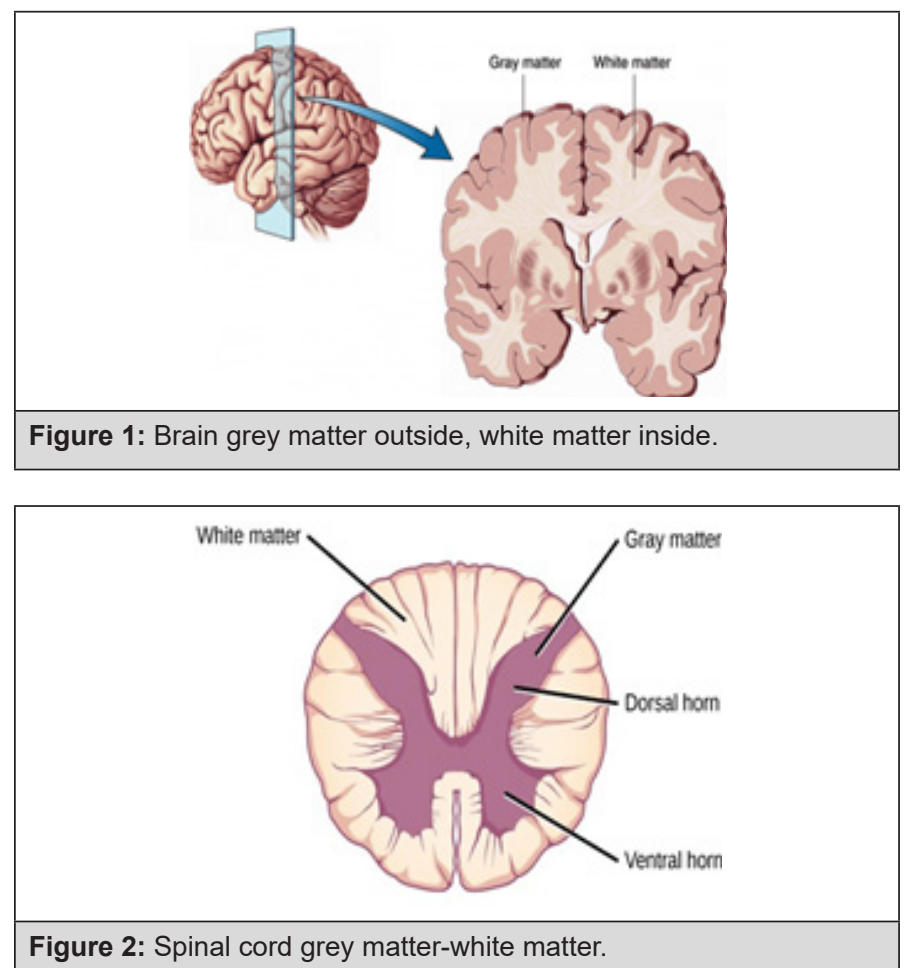

The brain and spinal cord are fully protected by bone structures (the skull and spine) and by three outer membranes or meninges:

a. Dura Mater - outermost, thick and fibrous;

b. Arachnoid - has a vascularization that resembles a spider web;

c. Pia Mater - the most internal, thin and adherent to the CNS.

Below the arachnoid is the cerebrospinal fluid or cerebrospinal fluid, which has a protective function, involving the entire CNS.

The spinal cord does not fully occupy the vertebral canal. It ends at about the level of the 1st or 2nd lumbar vertebra. Spinal anesthesia, which worries so many people, offers no danger of traumatizing the spinal cord because it is made with a needle that penetrates the spinal canal below the 2 nd lumbar vertebra. Below this level is only one very thin fibrous cord - the filum Terminable that attaches the lower end of the medulla to the coccyx. Thus, the marrow should always be stretched.

On its way, the spinal cord emits the spinal nerves, always in pairs. And you may notice that these nerves are closely related to gray matter.

Many reflex acts are controlled directly by the spinal cord without brain interference. But in most cases, nerve stimuli reaching this organ are then transmitted to the brain, first reaching the diencephalon (region covering the hypothalamus) and then radiating to the most varied areas of the brain.

Is possible to verify that during vertebrate's evolution increased brain volume vs inferior vertebrates and this was responsible of the opposite architecture of brain vs spinal cord related white- grey matter anatomy.

The cortex is more focused in the new superior cognitive function's vs spinal cord

Whit more specialization in condition of the stimuli [2].

\section{The Peripheral Nervous System}

The PNS consists of the internal network of nerves that depart from the CNS and are distributed throughout the body (motor nerves) and from nerves that come from all areas of the body and converge on the CNS (sensory nerves). Of course, there are mixed nerves whose characteristics include those of all types mentioned above, that is, they carry all orders of the CNS to the various points of the body and at the same time transmit the sensory perceptions of those same points to the CNS.

We can then say that the PNS (peripheral nervous system) comprises all the nerves in our body. Many of these nerves act on the will of the individual, revealing voluntary action. These voluntary action motor nerves, along with the sensory nerves (which allow us to see, hear, feel pain, smell, taste, heat or cold etc.), offer the individual the possibility to relate to the environment. Therefore, they form what we may call the nervous system of relationship life. This system contrasts with another large number of nerves that act without the individual's conscience or will, regulating the activity of numerous organs such as the heart, stomach, intestines, diaphragm movements, salivary gland secretions, the pupil diameter etc. These involuntarily acting nerves, which work without one even suspecting, together form the autonomic nervous system or the vegetative life nervous system.

There are lesions that destroy areas of the CNS, completely nullifying the nervous system acting on the relationship life nervous system but leaving the nervous system of the vegetative life intact. When this occurs, the person becomes unrelated to the world around him and goes on to live an extremely vegetative life 
(the organs work well, but the individual seems to feel nothing or respond to external stimuli).

It is common to call the nervous system of life a somatic nervous system relation (from the Greek soma, "body"), which does not seem very logical to us, since the autonomic nervous system, acting on the various parts of the body, is, consequently also somatic.

The nervous system of relationship life comprises nerves that originate directly in the brain (particularly, the brain, cerebellum, pons or bulge, and, more numerously, the bulb) and nerves that originate in the spinal cord. We then distinguished cranial and spinal nerves, respectively.

Cranial nerves are those that are born directly from the brain. In mammals they number 12 pairs (in other vertebrates there are only 10 pairs). Some are sensitive; others, engines; still others are mixed. All are cataloged by numbers. Often, a pair is referred to by its number, not by its name.

Thus, it is mandatory to know the 12 pairs of cranial nerves by their order numbers:

1. Olfactory (sensitive): It transmits to the brain the impulses that give the perception of smell.

2. Optical (sensitive): Brings to the brain the impulses that provide the visual sensations (Figure 3).

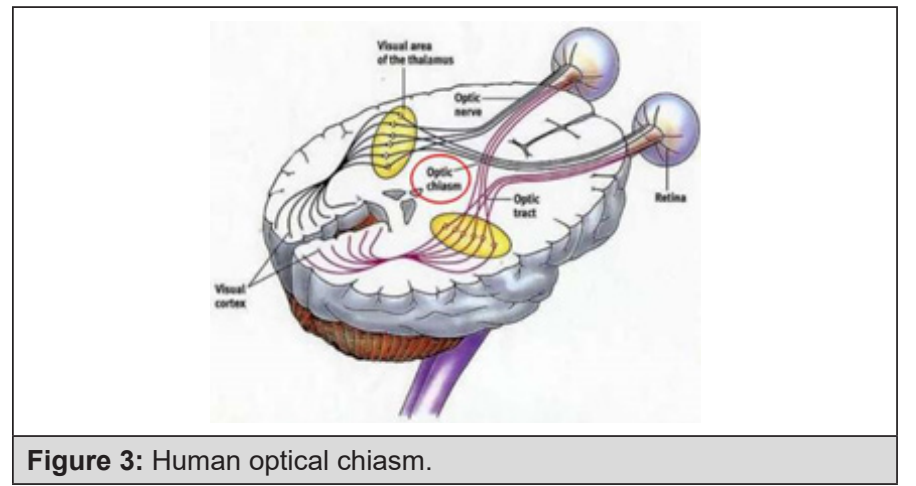

3. Common Eye Engine or Oculomotor (engine): Moves the eyes up, down and in (nose direction).

4. Pathetic or Trochlear (motor): Makes the eyes rotate circularly.

5. Trigeminal (mixed): Perceives sensations of the face and acts on the muscles of the mimic.

6. Outboard Eye Engine or Abdulent (motor): Moves the eyes outward.

7. Facial (mixed): It transmits the skin sensations of the face and acts in mimicry.

8. Acoustic or Atrio-cochlear (sensitive): One of its branches leads to the brain impulses that will give sound perceptions. The other leads to the cerebellum impulses responsible for the notion of body balance.
9. Glossopharyngeal (mixed): Transmits the impulses that give the perception of taste and moves the tongue.

10. Pneumogastric or Vagus (mixed): It acts on the thoracic and abdominal organs and is the main nerve of the parasympathetic system.

11. Spinal, Spinal or Accessory (motor): Acts on the shoulder muscles (shoulder slapping of the naughty).

\section{Hypoglossus (motor):}

Helps glossopharyngeal in tongue movement.

They all act on organs and muscles from head to shoulder. Only the pneumogastric or vagus goes into the body and innervates the viscera, such as the heart, stomach, intestines and other organs. In fact, this is the only cranial pair that has involuntary action, therefore belonging to the autonomic nervous system.

The spinal nerves are all born from the spinal cord, but they go to different parts of the body, such as arms, trunks and legs. They comprise 31 pairs and are all mixed, that is, they transmit sensations of the skin and organs to the spinal cord, as they transmit its motor orders to the muscles.

Each spinal nerve contains sensory fibers, which bring to the medulla sensory perceptions of a region of the body, and motor fibers, which carry motor stimuli from the medulla to these regions. The spinal nerves emerge from the medulla through two roots anterior root and posterior root - which join just below to form the nerve itself.

Posterior roots (with sensory fibers) are afferent to the medulla, as they conduct the stimulus towards it. The anterior roots (with motor fibers) are efferent in relation to the medulla, because they carry stimuli that move away from it.

To its greatest extent, therefore, each spinal nerve encloses sensory and motor fibers and proceeds as a "two-way road". From the sensory fibers come the perceptual stimuli and from the motor fibers the command commands.

Transition (or association) neurons can make the connection between a sensory neuron and a motor neuron:

A. on the same side and at the same level as the gray matter of the medulla;

B. opposite but at the same level in the medulla (horizontal cross-association);

C. on the opposite -side and at another level in the medulla (vertical cross association);

D. on the same side and at another level (uncrossed vertical association).

Surely you have ever touched a finger harder than you expected on the tip of a needle. And he withdrew his finger abruptly, so quickly 
that it would not be possible or consciously for him. This fact is an example of arc reflex. The reflex arc is the immediate response to arousal of a nerve without the interference of the individual's will (and sometimes even consciousness).

In the above example, the stimulus ran through the sensory fibers of a spinal nerve, bypassed the gray matter of the spinal cord by the association neuron, and returned through the motor fibers of the spinal nerve, reaching the muscles of the arm and hand, causing them to contract. and remove the finger from the tip of the needle.

Many reflexes are by medullary mechanism only. The rotulian or patellar reflex, which the doctor investigates a small blow to the rotulian tendon (knee), denotes through the sudden response of the musculature, involuntarily kicking the air, that the spinal nerves of this region, as well as the medulla, are perfect and in good working order.

But some reflexes are more complex and involve stimuli that go to and return to the cerebral cortex bringing orders to the marrow.

From the analysis of the figure above, you can see:

At the level of the bulb, stimuli from one side of the body transfer to the opposite -side of the brain, just as motors coming from one cerebral hemisphere cross at the level of the bulb or medulla. Across the body in a reflex involving the medulla and the brain, the sensory stimulus moves back and forth at the level of the bulb, but the motor response coming from the brain only reverts to the primitive side at the level of the medulla (Figure 4) [3].

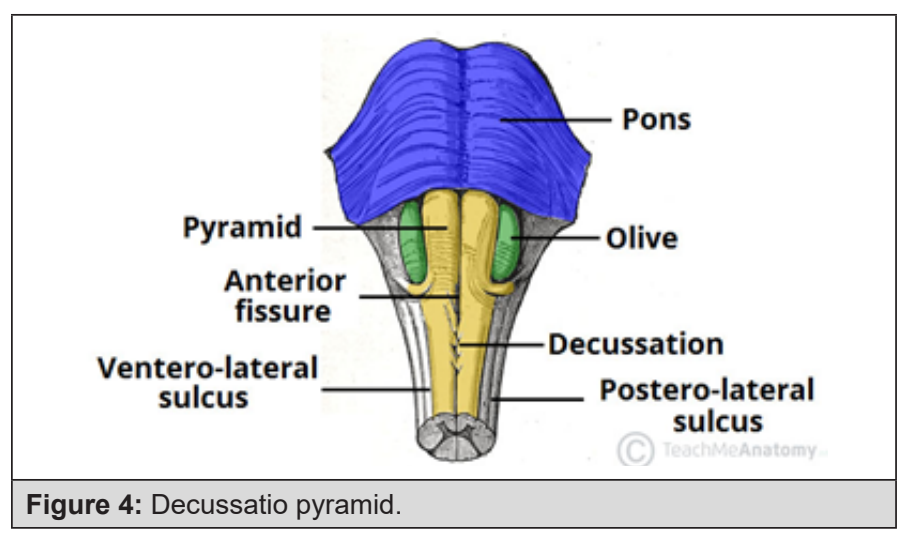

\section{The Autonomic Nervous System}

The autonomic nervous system or neurovegetative system is formed by nerves that work without any dependence on the will or conscience of the individual. These nerves are divided into two large groups: the sympathetic system and the parasympathetic system. Both groups are antagonistic. In the organ in which the sympathetic nerves act by stimulating, the parasympathetic nerves that will have their act inhibiting. In other organs, the parasympathetic is exciting and the sympathetic is inhibiting.

The heart is stimulated by the sympathetic hair and inhibited by the parasympathetic. The opposite occurs, however, in the gut.
From the antagonism of both groups, the functional balance of the organism arises. When one of the groups becomes disturbed, there is imbalance, and the organic functions begin to disturb. This explains neurovegetative disorders such as tachycardias (palpitations), vomiting, diarrhea, changes in blood pressure, salivation, etc.

The nerves of the sympathetic system are all born from anteriorbranches of spinal nerves SPN. The nerves of the parasympathetic system form, some also from anterior branches of spinal nerves, while others are born directly from the brain (these are the various branches of the pneumogastric or vagus).

The nerves that make up the ANS (autonomic nervous system) end in glands or smooth muscle tissue. They command, without the individual's conscience, the secretory activity and movements of the viscera in whose structure there are smooth muscles.

Sympathetic parasympathetic Pupils dilate contracts Heart rate accelerates (tachycardia) Retards (bradycardia) Blood vessels contract (person becomes pale) Dilates (person turns red) Stomach paralyzes excites Intestine paralyzes excites Bladder relaxes contracts.

The nerves of the anastomoses sympathetic system gather their roots and form the sympathetic plexuses, such as the cardiac plexus (which acts on the heart), the solar plexus or celiac plexus (whose branches will form in the liver, stomach and intestines). sacral plexus (which acts on the urogenital organs). All these organs also receive nerve branches from the parasympathetic system.

Neural development is one of the earliest systems to begin and the last to be completed after birth. This development generates the most complex structure in the embryo and the long time period of development means in utero insult during pregnancy may have consequences to development of the nervous system.

\section{Related Human Embryology}

The early CNS begins as a simple neural- plate that folds to form a neural groove and then the neural- tube. This early neural is initially open at each end forming the neuro-pores. Within the neural -tube stem cells generate the 2 major classes of cells that make most of the NS: neurons and glia. This cell differentiates into many different types generated with highly specialized functions and shapes [4-6].

\section{Material and Methods}

After this introduction whit a bibliographic research some relevant literature reported in references is analyzed in order to produce a global conclusion.

All literature was founded on PUBMED database.

\section{Results}

Comparing the information reported in the introduction section whit some relevant literature related some human 
neurodegenerative condition is clear that compared anatomy and physiology is a useful instrument for base research and to produce new pharmacological strategy.

\section{Literature}

\section{a. Quan Wen et al. [7]}

"A ubiquitous feature of the vertebrate anatomy is the segregation of the brain into white and gray matter. If evolution maximized brain- functionality, what is the reason for such segregation? To answer this, we posit that brain functionality requires high inter-connectivity and short conduction delays. Based on this assumption we re-searched for the optimal brain architecture by comparing different candidate designs. We found that the optimal design depends on the number of neurons, interneuronal connectivity, and axon diameter. the requirement to connect neurons with many fast axons drives the segregation of the brain into white and gray matter. These results provide a possible explanation for the structure of various regions of the vertebrate brain, such as the mammalian neo-cortex and neo-striatum, the avian tele-encephalon, and the spinal cord" [7].

\section{b. Carlos Matute et al. [8]}

"The phylogenetic enlargement of cerebral cortex culminating in the human brain imposed greater communication needs that have been met by the massive expansion of WM (white matter), as opposed to the GM (grey matter), exclusively contains axons and their glial cell partners; absent from WM are neuronal cell bodies, dendrites and conventional synaptic structures. Glial cells in WM are unique. WM astrocytes have especially long, highly discrete processes, which have led to their designation as 'fibrous' astrocytes. Oligo-centrocytes, which make and sustain myelin, predominate in $\mathrm{WM}$, although their density varies regionally as a function of the percentage of axons that are myelinated in a given tract (e.g. 100\% in optic nerve to fewer in corpus callosum). Myelin consists of tightly wrapped oligo-dendrocytic processes that surround larger diameter axons and mediate saltatory action potential conduction, which increases conduction velocity by at least 50 -fold compared with un-myelinatedfibres of similar diameter" [8].

\section{c. Giulio Srubek Tomassy et al. [9]}

"The evolutionary success of the vertebrate NS is largely due to a unique structural feature - the myelin sheath, a fatty envelope that surrounds the axons of neurons. By increasing the speed by which electrical signals travel along axons, myelin facilitates neuronalcommunication between the distant regions of the nervous system Myelin evolution.

As animals populated different and more challenging environments, the rapid conduction of the nerve impulses must have constituted a great adaptive advantage, enhancing chances of survival for both predators and prey. Neuronal impulse conduction is modeled as a flow of ions through a hollow cylinder. In this model, 2 physical parameters of the cylinder critically affect the speed of ion flow: axial resistance and capacitance of the surface. Through alterations in either or both 2 parameters, different evolutionary strategies achieved the same goal - increasing the conduction velocity of neuronal signals to make "faster" NS. In some species, axonal diameter was increased in order to decrease the axonal internal resistance and thus speed up signal conduction, an adaptation which resulted in the 'giant axons' of many invertebrates; in vertebrates, development of the myelin sheath increased the axial resistance of the axonal surface in addition to reducing its capacitance.

Robust evidence exists for the evolutionary- advantage that myelin provides. Myelin repeatedly emerges among species that are phylo-genetically distally unrelated; myelin-like structures are found even in some in-vertebrates, like members of the subphylum Crustacea (some decapods and copepods) and phylum Annelida (e.g. earthworms). Among myelinated invertebrate species, the structure of the myelin envelope can vary considerably from a loose arrangement of lamellae (e.g. in some decapods) to a "vertebratelike" compact architecture comprising tightly associated layers (earthworms). Bio-chemical properties, such as content and ratio of proteins and lipids within the myelin envelope, can vary among invertebrate species. In an even more distant example of evolutionary diversity, myelin-like structures with no glial origin were recently described in the nervous system of the copepod Bestiolinasimilis.

Myelin found around the axons of gnathostome vertebrates is more homogeneous and structurally similar, even between CNS and PNS, with fine differences observed only at higher magnification. Myelin is present in all vertebrates, from cartilaginous fishes to mammals, with the exception of the class Agnatha, jawless fish; for this reason, it has been hypothesized that appearance of myelin was concomitant with the appearance of a hinged jaw and that first myelinated gnathostomes may have been the placoderms (among the first jawed fish), whereas other jawless fish (e.g. ostracoderms) may have not been myelinated.

Whether loose or compact, produced by Schwann cells, oligodendrocytes, and by yet-to-be-defined mechanisms, myelin is a perfect example of convergent evolution. By increasing the speed of impulse conduction, myelin certainly contributed to the expansion of the vertebrate brain and to the emergence of complex- plastic behaviors "[9].

\section{d. Michel A Hofman [10]}

"The most obvious problem imposed by large brains is increasing distances among the neuronal somata of functionally related regions and the inevitable lengthening of their essential communication lines, the axons. the axonal length and volume increase much more rapidly than the number of neurons. a proportional increase of neurons and connections would inevitably lead to a rapid increase of synaptic path length, defined as the average number of mono-synaptic connections in the shortest path between two neurons So that the path length can be maintained, 
short cut connections can be inserted, resulting in small-world- and scale-free-type networks.

Although such a solution can effectively decrease path length within the neo-cortex, the increased lengths of the axons and the associated increased travel time of the action potentials still pose serious problems. As compensation for these excessive delays, axon caliber and myelination should be increased .An indication that larger brains deploy both more shortcuts (long-range connections) and larger-caliber axons is that the volume of the white matter increased at $4 / 3$ power of the volume of gray matter during the course of evolution. Although the white matter occupies only $6 \%$ of the neo-cortical volume in hedgehogs, it exceeds $40 \%$ in humans" [10].

\section{e. Cassandra Sampaio-Baptista et al. [11]}

"Recent evidence suggests that oligo-dendrocytes can form compact myelin sheaths even in the absence of molecular axonal cues, and that sheath length depends not on properties of the fiber but on the regional origin of the oligo-dendrocyte (brain versus spinal cord "[11].

\section{f. Marc R Freeman et al. [12]}

"III. Evolution of Brain Complexity: More and Diversified Glia Is, Evidently, Better

In-vertebrate glia carry out many functions that are analogous to their vertebrate counterparts. The Drosophila nervous system comprises about 105 neurons compared to $85 \times 106$ neurons in the human brain. Glia make up about $15 \%$ of the C. elegans and Drosophila nervous systems, but estimates range from $50 \%-90 \%$ of cells in the human brain, implying that greater glial numbers were essential for achieving increased brain complexity. The increased size of the brain required new mechanisms for proliferation and expansion of glial pool size and long-range conduction across white matter tracts. Beyond just increasing numbers, glia may also have acquired enhanced functions and diversity. Cell-intrinsic morphological and functional differences have been observed within mammals between mouse and human astrocytes. Other examples of enhanced glial functions are below.

Strategies to Enhance Nerve Conduction.

Selective pressure for more rapid conduction of the nervous impulse, e.g., in escape or attack behaviors, increasing brain complexity, etc., resulted in 2 types of solutions: decreasing longitudinal resistance or increasing capacitance of axons. Invertebrates have unsheathing cells but generally lack myelin. Exceptions are earthworms, copepods, and some crustacean nerves, but myelin and organized white matter tract, as such, are generally found only in vertebrates above the jawless fishes. In non-myelinated axons, velocity of the action potential is directly proportional to the axon diameter. The major conduction speed augmentation strategy in in-vertebrates is reducing longitudinal resistance by increasing the diameter of axons. Prime examples of this are found in cephalopods that accommodate a very large diameter axon or the Drosophila giant fiber, which drives the escape response.

Vertebrates have other constraints that place limits on using this strategy, including limiting bony structures, greater size requiring longer axonal lengths in the CNS and PNS, and with increasing brain complexity there is the need to pack many more axons in each space. The solution for accommodating many small-diameter axons is to reduce the effective capacitance and increase the effective membrane resistance, which is achieved by providing a layer of insulation, which is achieved with myelination. Myelin sheathes also organize sodium channels into clusters (nodes of Ranvier) for saltatory (jumping) conduction. For an axon of equivalent diameter, myelin can increase the velocity of nervous impulse conduction by 50 - to 100 -fold. It should also be noted that oligo-dendrocytes carry out other functions in support of axon integrity, likely an adaptation brought about to deal with energy and trophic demands of the extraordinarily long fast-firing axons found in many higher organisms. a recent study showed that deficiency of a lactate transporter in oligo-dendrocytes led to axono-pathy and degeneration "[12].

\section{g. Suzana Herculano-Houzel et al. [13]}

"Scaling of ratios of neurons over the rest of brain

The spinal cord and brain-stem are the portions of the CNS that are most directly related to the regulation of bodily functions, and thus could be expected to scale in close relationship to the scaling of body physiology in its various aspects. Neurons in the cerebral cortex and the cerebellum, in contrast, are believed to add a whole new level of elaboration to the processing of information relayed from the body and back to it through associative processing, endowing animals with more refined and flexible behavioral repertoires.

In the absence of data on numbers of neurons and volumetric data for the spinal cord, the ratio of cortical volume over the volume of the medulla has been proposed as a value that should predict cognitive capacity in a manner that is not biased by body mass Variations in this ratio across primate species indeed were well correlated with available behavioral data, but so were brain size, relative cortical volume and encephalization quotient . that comparison assumed that the volumes of the cerebral cortex and of the medulla are good proxies for numbers of neurons in the structures, whereas we have shown that this is not the case across clades. the ratio between numbers of neurons in the cerebral cortex and in the brainstem, or spinal cord, might provide a good estimate of how cortical processing capacity scales beyond body-related information processing across species.

Across primate species, we found that numbers of neurons in the spinal cord are linearly related to the length of the spinal cord, not body mass the cerebral cortex gains neurons as a power 
function of numbers of neurons in the spinal cord with exponent 2 , even though the mass of the cerebral cortex (including white matter) scales only slightly faster than the mass of the spinal cord, as a power function of exponent 1.

Un-fortunately, data on total numbers of neurons in the spinal cord that can be compared to numbers of neurons in the brain are only available for primates. we found in that study that the number of neurons in the ensemble of brainstem, diencephalon and striatum, which we refer to as "rest of brain", scales linearly with the number of neurons in the primate spinal cord. This linearity warrants the use of numbers of neurons in the rest of brain, which are available for all 41 species in our sample, as a proxy for numbers of neurons in the spinal cord and also for the increase in numbers of neurons that would be directly related to any variations in body size (regardless of whether total volume, sensory surface area, muscular mass or energetic requirement is the relevant parameter). We thus use numbers of neurons in these structures as an internal reference for the examination of how information processing might scale faster in the cerebral cortex and in the cerebellum than required for dealing strictly with bodily functions, without having body mass as a confounding variable" [13].

"the cerebral white matter, which contains not only axons but around 2 billion neurons and a large, but unknown, number of glia in humans, seems to increase dis-proportionately compared to gray matter as brain size scales across species. Not surprisingly, the cerebral neo-cortex and cerebellum, which contain the largest amounts of white matter, tend to make up greater proportions of larger mammalian brains" [14].

\section{h. Harvey J Karten [15]}

"Progressive Telecephalization of Function

By the end of the nineteenth century, Herrick et al had demonstrated that the brain-stem of all vertebrates shared a profound level of similarity. the thalamus and telencephalon, except for the olfactory bulbs, seemed to show few commonalities between mammals and non-mammalian vertebrates. This led to the prevailing view that the forebrain of most non-mammalian vertebrates was related to olfactory inputs. The mammalian forebrain, particularly the cortex of the telen-cephalon, was increasingly thought to be novel and unique to mammals. There was no structure in the non-mammalian forebrain that could readily be compared with the mammalian cortex. The belief in the uniqueness of the mammalian forebrain was particularly emphasized in the writings of Sir HJackson (1911), and his co-worker, D.Ferrier (1928), who suggested that over the course of evolution, functions of the brainstem were transferred to the forebrain. This was referred to as the progressive tele-encephalization of complex functions. Examples of such functions included the ability to decode auditory inputs generated by vocal communication, visual pattern recognition, visual stereopsis, deciphering complex somato-sensory inputs and most notably, so-called higher cognitive functions. The level of analysis performed was judged to be that requiring the participation of the neo-cortex in mammals.

But how could non-mammalia perform such operations in the absence of modal-specific thalamic nuclei and cortical regions? Structures within the forebrain, such as the specific sensory relay nuclei of the thalamus and the 'neo-cortex' of the telencephalon, were largely considered unique to mammalian brains. The telencephalae of non-mammalia were considered to consist almost exclusively of olfactory centres and basal ganglia. This directly implied the lack of refined lemniscal visual, vestibular, gustatory, auditory or somatosensory inputs to the tele-encephalon, and certainly no prospect of ability to deal with discrete stimuli from any of these sources. It also posed a paradox among birds, as many species of birds with large tele-encephalae have only very limited, or no olfactory capabilities, particularly when compared with many non-avian reptiles and mammals. What might be the possible function of the large avian tele-encephalon?

the notion of the uniqueness of mammals with a distinct thalamus and neo-cortex was based on painfully sparse information. The afferent connections to the thalamus in non-mammalian brains, their projections upon the telencephalon and the various discrete populations of the telencephalon were almost totally unexplored. This led to the erroneous notions that the thalamic and cortical populations of the mammalian brain were unique to mammals and arose abruptly with the evolutionary origin of mammals "[15].

Nat Rev Neurosci. Author manuscript; available in PMC 2008 Aug 12.

\section{i. Jarvis ED et al. [16]}

"They noted that the main divisions of the human CNS-the spinal cord, hindbrain, midbrain, thalamus, cerebellum and cerebrum or tele-encephalon-were present in all vertebrates. Edinger, however, noted that the internal organization of the telencephala showed the most pronounced differences between species. In mammals, the outer part of the telencephalon was found to have prominently layered grey matter whereas the inner part had nuclear grey matter. The inner part was located ventrally to the lateral ventricle. The outer part was more elaborate and folded in humans than in smaller- mammals. In non-mammals, the outer and inner parts of the tele-encephala were mainly composed of nuclear grey matter, most of which was located ventrally to the lateral ventricle in reptiles and birds On the basis of these considerations, Edinger proposed that tele-encephalic evolution occurred in progressive stages of increasing complexity and size, culminating with the human cerebrum. He suggested that the stages proceeded in a ventral-to-dorsal direction, with each new vertebrate group acquiring a more advanced cerebral sub-division, much as the earth's geological strata formed over time. He proposed that, first, there was the old brain, the palaeo-encephalon (also called the basal ganglia or subpallium at the telencephalic base), which controlled instinctive behaviour, followed by the addition of a new 
brain, the neo-encephalon (also called the pallium or mantle at the top of the tele-ecephalon), which controlled learned and intelligent behaviour. He, A.Kappers and others named the tele-encephalic subdivisions within each vertebrate group with the prefixes 'palaeo' (oldest),'archi' (archaic) and 'neo' (new) to designate the presumed relative order of evolutionary appearance of each subdivision. In Greek, 'archi' means the oldest, the first, or the most primitive, whereas 'palaeo' meansancient, primitive or old, but not necessarily the oldest. Both Edinger and AriënsKappers misinterpreted the meaning of these prefixes and reversed them, naming structures with 'palaeo-' to indicate the oldest or first and 'archi-' to indicate old. They added to these prefixes the root word 'striatum' for the presumed palaeo-encephalic subdivisions and 'pallium' or 'cortex' for the presumed neoencephalic subdivisions. The term 'striatum' was used because a large part of the basal ganglia (palaeo-encephalon) in mammals, now commonly called the caudate-putamen, has fibre bundles coursing through it that give it a striated appearance.

The classical view that became dominant was that the primordial telencephalon of fishes had a relatively small pallium and a larger sub-pallium, both of which were entirely devoted to olfactory information processing. The fish sub-pallium was named 'palaeostriatum' (old striatum) and was thought to be the antecedent of the human Globus pallidus. Amphibians were thought to have evolved an 'archi-striatum' (archaic striatum) above the palaeostriatum, which was proposed to be the antecedent of the human amygdala. Reptiles were thought to have evolved a 'neo-striatum' (new striatum) above the archi-striatum, which was proposed to be the antecedent of the human caudate and putamen. The palaeostriatum of reptiles was also thought to have elaborated into an older part (primitivum) and a newer part (augmentatum), both of which were considered homologous to the human globuspallidus. Following this, birds were thought to have evolved a large additional basal ganglia subdivision, the 'hyperstriatum' (hypertrophied striatum), which was considered to be unique to birds.

The fish pallium was named 'palaeo-cortex' and was proposed to be the antecedent of the human olfactory cortex. Reptiles were thought to have evolved an 'archi-cortex', also thought to be olfactory and primitive, that was said to be the antecedent of the human hippocampus. Birds were thought not to have evolved any further pallial regions. By contrast, mammals were thought to have evolved the latest and greatest achievement, a 'neocortex', from the palaeocortex and/or archicortex6. The archi-cortex and/or palaeocortex, with their 2-3 cell layers, were assumed to be primitive; the neocortex, with its 6 layers, was assumed to be more recently evolved and a substrate for more sophisticated behavior.

There were dissenting voices to the classical view. Some of its proponents also made partial or tentative retractions. alternative views were not widely embraced. Instead, the classical view was codified in the important 1936 comparative neuro-anatomy text by
A Kapperse et al and became pervasive throughout neuroscience. A new view of telencephalic evolution.

With this new understanding of the avian telencephalic organization and its homologies with that of mammals, we can generate more informed hypotheses and conclusions about telencephalic evolution in vertebrates. It is now apparent that the organization of the true basal ganglia among birds, mammals and other vertebrates (that is, distinct nuclear striatal and pallidal domains with more dopaminergic input into the striatal domain) is quite conserved. By contrast, the organization of the pallial domains of these groups is more varied. The avian hyperpallium has a unique organization that has so far been found only in birds. This consists of semi-layered subdivisions and might have evolved more recently than the mammalian six-layered cortex, as birds evolved 50-100 million years after mammals. The DVR (which, in birds, contains the meso-pallium, nido-pallium and arco-pallium) is a nuclear, grey matter formation that is unique to birds and reptiles. The six-layered cortex is unique to mammals, and, as all the main groups of living mammals (monotremes, marsupials and placentals) have a six-layered cortex87, it was presumably inherited from their common therapsid ancestor more than 200 million years ago. Furthermore, new findings indicate that mammals did not arise from reptiles, but from therapsids, and that the last common ancestor of the reptile and mammal lineages was the stem amniotes. As all non-mammalian therapsids are now extinct, it is difficult to trace from stem amniotes to mammals the evolutionary history of mammalian tele-encephalic organizationlayered, nuclear or otherwise. Therefore, the reptilian nuclear pallial organization cannot be assumed to represent the ancestral condition for mammals "[16].

\section{j. Fahima Mayer et al. [17]}

Pharmacologic remedy of many brain diseases is difficult because of the powerful drug exclusion properties of the bloodbrain barrier (BBB). Chemical isolation of the vertebrate brain is achieved through the highly integrated, anatomically compact and functionally overlapping chemical isolation processes of the BBB. These include functions that need to be coordinated between tight diffusion junctions and uni-directionally acting xenobiotic transporters. Understanding of many of these processes has been hampered, because they are not well mimicked by ex vivo models of the BBB and have been experimentally difficult and expensive to disentangle in intact rodent models. Here we show that the Drosophila melanogaster (Dm) humoral/CNS barrier conserves the xenobiotic exclusion properties found in the vertebrate vascular endothelium. We characterize a fly ATP binding cassette (ABC) transporter, Mdr65, that functions similarly to mammalian xenobiotic BBB transporters and show that varying its levels solely in the Dm BBB changes the inherent sensitivity of the barrier to cytotoxic pharmaceuticals. Furthermore, we demonstrate orthologous function between Mdr65 and vertebrate $\mathrm{ABC}$ 
transporters by rescuing chemical protection of the Dm brain with human MDR1/Pgp. These data indicate that the ancient origins of CNS chemoprotection extend to both conserved molecular means and functionally analogous anatomic spaces that together promote CNS selective drug partition. Thus, Dm presents an experimentally tractable system for analyzing physiological properties of the BBB in an intact organism.

In vertebrates, a physically separate blood-brain barrier (BBB), primarily engineered into the single-cell layer vascular endo-thelium (VE), provides anobstacle to chemical attack. At this interface, strong selective pressures have produced the integration of at least two very different cell biologic mechanisms to prevent free movement of small molecules between the humoral and CNS interstitial compartments. BBB VE cells impede the traffic of drugs by virtue of specialized lateral junction components, including tight junctions, and asymmetrically arrayed ATP binding cassette (ABC) transporters. Tight junctions prevent para-cellular diffusion of charged molecules, and asymmetrically arrayed transporters actively expel lipophilic molecules backinto the humoral space. Together, these complimentary systems prevent the majority of xeno-biotics from acting on vertebrate nervous tissue. Although in vivo and in vitro BBB models have confirmed the importance of these two components, substantial limitations hinder progress. A powerful BBB model system should combine molecular genetics, genomic, chemical biology, and integrative physiology tools to probe CNS-specific chemo-protective physiology. For this, we turned to Drosophila melanogaster (Dm) and asked what aspects of BBB physiology can be modeled in an in-vertebrate.

Insects also possess protective neural barriers, but they differ anatomically from vertebrates. Dm has an open circulatory system that is separated from the CNS by a thin layer of glially derived epithelial cells making the Dmhumoral/CNS interface topologically much simpler than the vertebrate BBB. on a cellular level, the vertebrate and insect BBBs share many common features. one specific cell layer of the Dm BBB, the subperineural glia (SPG), possesses elaborate laterally localized homotypic junctional complexes, or pleated septate junctions, that create a tight barrier to para-cellular diffusion The Dm proteins that make up the pleated septate junctions are nearly identical to the vertebrate proteins that compose the tight junctions .disruption of the pleated septate junctions leads to defects in Dm BBB function but the dual nature of localized xenobiotic protection mechanisms had not been established in insects" [17].

\section{k. Bufill E et al. [18]}

"Alzheimer's disease is a complex disease associated with advanced age whose causes are still not fully known. Approaching the disease from an evolutionary standpoint may help in understanding the root cause of human vulnerability to the disease. $\mathrm{AD}$ is very common in humans and extremely un-common in other mammals, which suggests that the genetic changes under-lying the alterations in cerebral structure or function that have taken place over the course of the evolution of the genus Homo have left specific neurons in the human brain particularly vulnerable to factors which trigger the disease. Most of the genes whose mutation leads to $\mathrm{AD}$ are involved in synaptic plasticity. Evidence has also been found relating $\mathrm{AD}$ to neuronal oxidative stress. Neurons in certain association areas of the human brain retain juvenile characteristics into adulthood, such as the increased expression of genes related to synaptic activity and plasticity, incomplete myelination and elevated aerobic metabolism, which can cause an increase in oxidative stress in these neurons. Oxidative stress can cause myelin breakdown and epigenetic changes in the promoter region of genes related to synaptic plasticity, reducing their expression. These changes may in some cases induce hyper-phosphorylation of tau and $\beta$-amyloid deposits, which are characteristic of AD. The adaptation of humans to the cognitive niche probably required an increase in synaptic plasticity and activity and neuronal metabolism in neurons in areas related to certain cognitive functions such as autobiographical memory, social interaction and planning. The cost of these changes may have been the brain's increased vulnerability to factors which can trigger AD. This vulnerability may have resulted from the evolutionary legacies that have occurred over the course of the evolution of the human brain, making AD a possible example of antagonistic pleio-tropy. The evolutionary approach allows apparently unrelated data from different disciplines to be combined in a manner that may lead to an improved understanding of complex diseases such as Alzheimer's" [18].

\section{l. Mark R Cookson [19]}

"There are a number of neuro-degenerative diseases that principally affect humans as they age, characterized by the loss of specific groups of neurons in different brain regions. Although these are in general sporadic disorders, it is now clear that many of these diseases have a substantial genetic component. As genes are the raw material with which evolution works, we might benefit from understanding these genes in an evolutionary framework. Here, I will discuss how we can understand whether evolution has shaped genes involved in neurodegeneration and the implications for practical issues such as our choice of model systems for these diseases and more theoretical concerns such as the level of selection against these phenotypes.

Evolutionary theory, modified to include our modern molecular views on genetics, permeates all aspects of modern biology. Understanding human biology therefore incorporates acknowledgement of our genetic heritage shaped by the evolutionary forces that have led to humans occupying our current niche. And it should not be surprising that, as a major aspect of our biology, the near universal experience of human disease can also be viewed through the evolutionist's prism.

I will discuss the relationship of evolution to age-related neurodegenerative disorders. This group of diseases is characterized by 
the shared property in the progressive loss of relatively specific groups of neurons. What distinguishes each is that during the aging process different groups of neurons are lost in each disease and these correlate with different clinical features. neuron loss in the hippocampus and cerebral cortex underlies many of the memory problems associated with $\mathrm{AD}$ whereas ataxia is a consequence of loss of Purkinje cells in the cerebellum and is characteristic of the spino-cerebellar ataxias. These symptoms are often profoundly disabling and sometimes fatal; loss of the neurons that innervate the diaphragm in amyotrophic lateral sclerosis (ALS) leads to an inability to breathe.

in many but not all neurodegenerative conditions, there are other pathological events including the accumulation of specific proteins in those neurons that survive. Often these are aggregated and insoluble and, more importantly, often the genes that code for these pathological proteins either cause inherited forms of disease and/or act as genetic risk factors. pathology, clinical phenotype and causal variation in specific genes are linked.

There are two questions to discuss here to understand the genes and proteins associated with neurodegeneration in the context of evolution. The first is in what ways can we use evolutionary views on sequences to understand aspects of protein function and dysfunction related to mutations associated with neurodegenerative diseases. This has practical implications, for example in assigning pathogenicity to specific mutations in genes or for understanding how far we can extrapolate from model systems to human diseases. The second, consideration is whether evolutionary forces have shaped these degenerative diseases. To provide a detailed example, I will first cover Parkinson's disease, a disorder that illustrates many of the key points under discussion here. I admit this is biased by my own research interests, so refer the interested reader to other review articles about the genetics of neuro-degenerative diseases more generally" [19].

DETOXING FOR BRAIN HEALTH - NEW RESEARCH FINDINGS: Craniosacral Therapy Improves Glymphatic Cleansing of Brain Tissue

\section{m. Carolyn Simon}

"New research provides evidence the body has a fast-track brain cleansing system that prevents diseases such as Alzheimer's and maintains brain health. Finding ways to support and enhance this cleansing process could lead to improved outcomes in brain injury and brain disease. Read on to find what scientists have discovered and how cranio-sacral therapy effectively promotes brain health by invigorating this active fluid -cleansing system.

Most of us have heard of the lymphatic system, the collection of vessels and nodes running throughout the body that helps cleanse waste products and is part of the body's immune system. Now a team of neuroscientists at the Univ. of Rochester Medical Center has identified a fascinating fast-track cleansing system in the brain called the glymphatic system. A bulk flow process moves CSF via the arterial system right into the brain tissue, exchanging with the interstitial fluid inside the brain. As it does, it washes through the tissue collecting waste particles that are sitting in between the brain cells. The CSF then enters the venous system via veins within the brain tissue, taking the fluid and the waste it picks up away from the brain. In this way waste material is efficiently removed from the brain tissue, by the CSF, via the circulatory system. We know accumulation of waste and toxic matter in the brain environment adversely affects brain- function".

Form an Article published in Scientific American https://www. scientificamerican.com/article/brain-cleaning-discovery/

Brain's Drain: Neuroscientists Discover Cranial Cleansing System

"Fluids coursing through the nervous system could help clear the brain of toxic detritus that leads to Alzheimer's and Huntington's disorders 2012.

The brain can be a messy place. Thankfully, it has good plumbing: Scientists have just discovered a cleansing river inside the brain, a fluid stream that might be enlisted to flush away the buildup of proteins associated with Alzheimer's, Huntington's and other neuro-degenerative disorders.

The researchers, based at the University of Rochester (U.R.), University of Oslo and Stony Brook University, describe this new system in the journal Science Translational Medicine today. The study adds to the evidence that the star-shaped cells called astrocytes play a leading role in keeping the nervous system in good working order.

In most of the body, a network of vessels carries lymph, a fluid that removes excess plasma, dead blood cells, debris and other waste. But the brain is different. Instead of lymph, the brain is bathed in cerebro-spinal fluid.

\section{n. Walker S Jackson [20]}

"The mechanisms under-lying the selective targeting of specific brain regions by different neuro-degenerative diseases is one of the most intriguing mysteries in medicine. it is known that A D primarily affects parts of the brain that play a role in memory, whereas PD predominantly affects parts of the brain that are involved in body movement. The reasons that other brain regions remain un-affected in these diseases are unknown [20] (Figure 5-8).

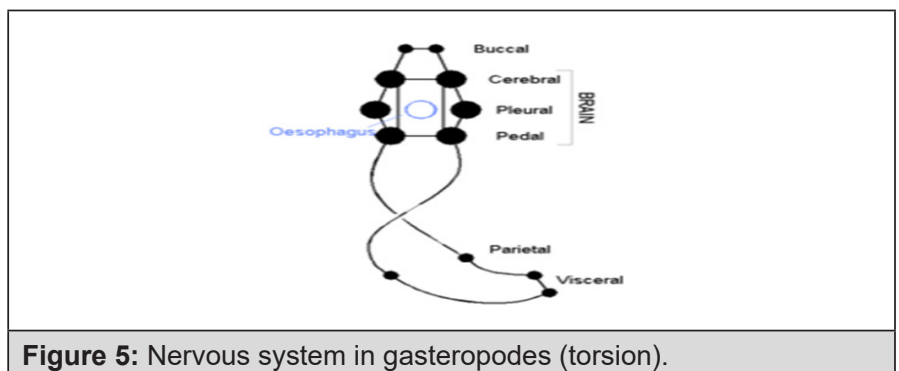



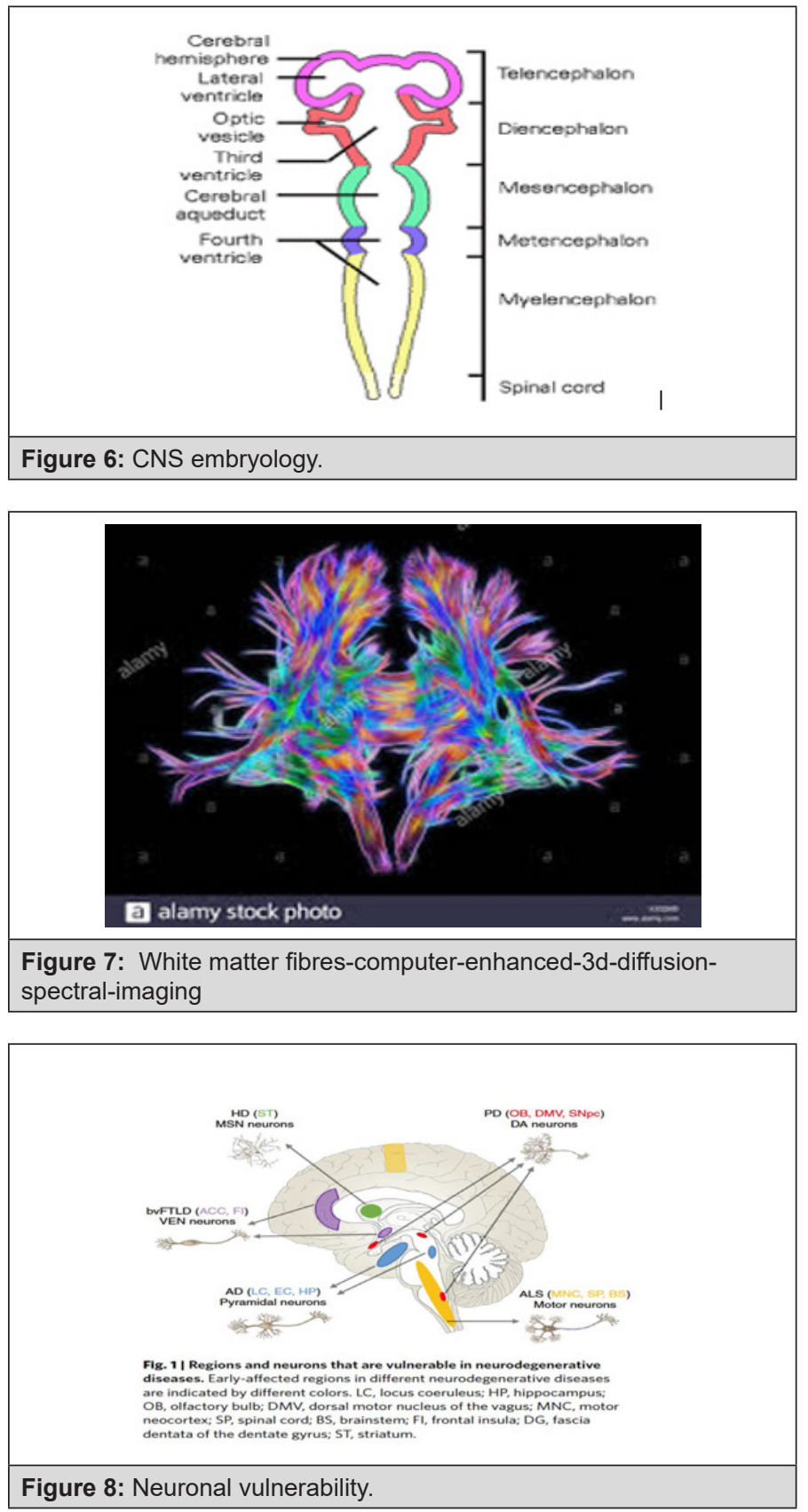

\section{Experimental project hypothesis:}

To verify the hypothesis that evolution pattern is involved in some neuronal vulnerability is possible to observe the incidence of cortical neuro degenerative pathology across the various superior vertebrates (in example related the increase of cortical cognitive advanced function) then compare this data also with the incidence of other degenerative process in inferior vertebrates.

\section{Discussion and Conclusion}

I. Is crucial in today research, related the actual LOW efficacy in some neurodegenerative disease, to deeply use compared anatomy and physiology as source of real interesting information.

II. The evolutive pattern as a key role in applied research.
III. The role played by phenomena like cephalization of nervous system, BEE, cortex development in vertebrates and other must be taken in great consideration when studying some neurodegenerative pathology.

IV. The development of new CNS STRUCTURES in evolutive patterns create a new

V. World of weakness in neuronal tissue. (see the differential vulnerability of different brain or spinal cord regions: this is a fact).

VI. Un-other fact is that DA is a common pathology not only in human but also in other.

VII. Superior vertebrates (cats, dogs).

VIII. The same other peculiarity is that in spinal cord or in brain different neurodegenerative disease are more often find DA more frequently involving the cortex cognitive function and ASL more involving the motorial system in spinal cord.

IX. Telencefalization evolution need to adapt the cortical expansion in the same skull dimension, and the characteristic presentation of grey and white matter in brain and in opposite way in spinal cord must be related to the global evolutive pattern of the NS in vertebrates.

X. Zoology, biology, anatomy, physiology, molecular biology, embryology, pharmacology as basic science for new Methodology in neurodegenerative disease research.

XI. The difference of NS of invertebrates (simpler) vs more complex on of vertebrates, related more complex motility need or cognitive perhaps make possible a peculiar neuronal vulnerability to be better studied.

\section{Clarifications}

This work is produced without any diagnostic or therapeutic intent, only to submit to researcher new hypothesis to be verified.

\section{References}

1. Mauro Luisetto, Farhan Ahmad Khan, Naseer Almukhtar, Ahmed Yesvi Rafa, Behzad Nili (2019) BOOK decussatio pyramid and optical chiasm as aninteresting example of evolutionary process useful in understanding some cervical spinal cord Phenomena.

2. Mauro Luisetto (2018) Journal of Neuroscience and Neurological Disorders Endogenous archeological sciences: Physiology, Neuroscience, Biochemistry, Immunology, Pharmacology, Oncology and Genetics as instrument for a new field of investigation.

3. Luisetto M, Ahmadabadi BN, Rafa AY, Sahu RK, Cabianca L, et al. (2019) The Turing machine theory for some spinal cord and brain condition, A toxicological-antidotic depurative approach. J Neurosci Neurol Disord 3: 102-134.

4. M Luisetto, Farhan Ahmad Khan, Luca Cabianca, Mobin Ibne Mokbul, Ahmed Yesvi Rafa (2016) Amygdala pharmacology and crime behavior, dysfunctions to be considered as a disease? International Archives of Biomedical and Clinical Research 2(2).

5. Luisetto M, Naseer Almukhtar, Farhan Ahmad Khan, Khaled Edbey, Ahmed Yesvi Rafa, et al. (2019) Mindset Kinetics and Crime Behavior- 
Quantitative Methods? A New Forensic Quantitative Approach. How Biochemistry, Toxicology, Imaging Principle can Help in Jurisdictional Settings. Am J Biomed Sci\& Res 2(1): 9-18.

6. Luisetto M, Gamal Abdul Hamid, Naseer Almukhtar, Ahmed Yesvi Rafa, Behzad Nili Ahmadabadi, et al. (2019) Molecular Characterization of Brain and other Structures in Vertebrates and Invertebrates. Arch Neurol\&Neurosci 3(5): 1-35.

7. Quan Wen, Dmitri B Chklovskii (2005) Segregation of the Brain into Gray and White Matter: A Design Minimizing Conduction Delays. PLoSComput Biol 1(7): e78.

8. Carlos Matute, Bruce R Ransom (2012) Roles of white matter in central nervous system pathophysiology. ASN Neuro 4(2): e00079.

9. Giulio Srubek Tomassy, Lori Bowe Dershowitz, Paola Arlotta (2016) Diversity matters: A revised guide to myelination. Trends Cell Biol 26(2): 135-147.

10. Michel A Hofman (2014) Evolution of the human brain: when bigger is better. Front Neuroanat 8: 15.

11. Cassandra Sampaio Baptista, Heidi Johansen-Berg (2017) White Matter Plasticity in the Adult Brain. Neuron 96(6): 1239-1251.

12. Marc R Freeman, David H Rowitch (2013) Evolving Concepts of Gliogenesis: A Look Way Back and Ahead to the Next 25 Years. Neuron 80(3): 613-623.

13. Suzana Herculano Houzel, Paul R Manger, Jon H Kaas (2014) Brain scaling in mammalian evolution as a consequence of concerted and mosaic changes in numbers of neurons and average neuronal cell size. Front Neuroanat 8: 77.

14. André MM Sousa, Kyle A Meyer, Gabriel Santpere, Forrest 0 Gulden, Nenad Sestan (2017) Evolution of the Human Nervous System Function, Structure, and Development. Cell 170(2): 226-247.

15. Harvey J Karten (2015) Vertebrate brains and evolutionary connectomics: on the origins of the mammalian 'neocortex'. Philos Trans R SocLond B Biol Sci 370(1684): 20150060.

16. The Avian Brain Nomenclature Consortium (2005) Avian brains and a new understanding of vertebrate brain evolution. Nat Rev Neurosci 6(2): 151-159.

17. Mayer F, Mayer N, Chinn L, Pinsonneault RL, Kroetz D, et al. (2009) Evolutionary Conservation of Vertebrate Blood-Brain Barrier Chemoprotective Mechanisms in Drosophila. J Neurosci 29(11): 35383550 .

18. Bufill E, Blesa R, Augustí J (2013) Alzheimer's disease: an evolutionary approach. J Anthropol Sci 91:135-57.

19. Mark R Cookson (2012) The evolution of neurodegeneration. Curr Biol 22(17): R753-R761.

20. Walker Jackson (2014) Selective vulnerability to neuro-degenerative disease: the curious case of Prion Protein. Disease Models \& Mechanisms 7(1): 21-29. 